grades (ST3-ST7). 75 respondents (33\%) intend to pursue subspecialty accreditation in hepatology. 103 respondents (46\%) have completed a period of training in a tertiary/transplant centre.

105 respondents (47\%) have worked in a centre where physicians perform liver biopsies. 52 (23\%) have been offered the opportunity to learn how to perform ultrasound assisted liver biopsy and 38 (17\%) have been assessed and deemed competent in the procedure. Three respondents commented that they are trained in unguided liver biopsy; one trainee has used the Sonosite probe and one commented that he/she has performed liver biopsy after an appropriate site was marked by the radiologist

137 (61\%) of trainees who responded to the survey would be interested in learning how to perform this procedure and a further $29(13 \%)$ may be interested. 138 (61\%) of respondents would be interested in attending a hands-on course to learn this procedure and 30 (13\%) may be interested. Of the 75 respondents who intend to pursue subspecialty hepatology accreditation, 72 said they would be interested in learning how to perform ultrasound assisted liver biopsy.

Trainees who participated in this survey reported that opportunities to learn this procedure were currently hit and miss. There were concerns about the practicality of maintaining competence during training and as a consultant and some respondents felt that it was safer for the procedure to be performed by radiologists.

We cannot report the views of trainees who did not complete the survey and therefore our results may not be representative. However even if we make the assumption that all non-responders are not interested in learning this procedure, there is still an estimated $15-20 \%$ of trainees that would be interested (137/ 800).

Conclusion Trainees participating in this survey are interested in learning to perform ultrasound-assisted liver biopsies, but the infrastructure to offer this training is not currently well established. Discussion between trainees and training bodies should be considered to explore this issue further.

Disclosure of Interest None Declared.

\section{PTU-007 DEVELOPMENT OF A SMARTPHONE APP TO AID THE CLINICAL MANAGEMENT OF POLYPOSIS SYNDROMES}

GH Lee*, A Latchford, RK Phillips, SK Clark. Polyposis Registry, St Mark's Hospital, Harrow, UK

\subsection{6/gutjnl-2014-307263.81}

Introduction Smartphone "apps" are becoming increasingly used by health care professionals (HCPs) as a quick and easy guide for delivering evidence-based medicine. "Apps" are particularly effective in providing guidelines accessible from a smartphone with contents that can be updated frequently. The Polyposis Registry at our institution has spearheaded the formulation of guidelines for the management of inherited polyposis syndromes. We set out to develop these into "app" form.
Methods Essential content of our institution's guidelines (based on published guidelines) was selected by a multidisciplinary team and edited to suitable format for the "app" programmers, and a trial version was produced. This was tested by a group of HCPs (colorectal surgeons, gastroenterologists, nurse specialists). A questionnaire was sent out after the trial to determine the usefulness and effectiveness of the "app".

Results Eighteen HCPs trialled the "app". 89\% found it relevant and useful in their clinical practice, and would use it at least once a month. $83 \%$ said that it provided the information they required, and all would recommend it to a colleague. None considered it hard to use. Some improvements were suggested, which will be implemented in the final version offered externally.

Conclusion We present an "app" which provides our evidencebased guidelines for the management of polyposis syndromes in an easily accessible and updatable form, and describe its development.

Disclosure of Interest None Declared.

\section{PTU-008 DEDICATED COLONOSCOPY TRAINING LISTS IMPROVE TRAINEE COMPLETION RATES TO MATCH A CONSULTANT BENCHMARK}

I Ewing*, J Lim, K Bryce, A Muddu. Gastroenterology, North Middlesex University Hospital, London, UK

\subsection{6/gutjnl-2014-307263.82}

Introduction Colonoscopy is the gold standard modality for investigation of colonic disease. ${ }^{1}$ The procedure can be challenging to perform. ${ }^{1}$ Complete colonoscopy, defined as intubation of the terminal ileum, neo-terminal ileum, or caecum, should be achieved in greater than $90 \%$ of cases on an intention to complete basis. ${ }^{1}$ Historically trainees have performed colonoscopy on service lists, and ad hoc training lists and may have had incomplete access to training. ${ }^{2}$ Trainees currently working in our unit perform colonoscopy on dedicated training lists prior to JAG certification of independence. We performed a large retrospective study of colonoscopy completion rate, comparing two groups of gastroenterology trainees with consultant Gastroenterologists.

Methods 5307 consecutive colonoscopies, from a five-year period in a single centre, were triaged by first endoscopist. Groups identified were 1) consultant Gastroenterologists 2) previous trainees (individuals who trained in the unit in the past, performing colonoscopy on service, adhoc training, and dedicated training lists) 3) Current trainees (employed in the unit at time of study, performing colonoscopy on dedicated training lists). Colonoscopy completion rate, as defined above, was determined for each group. Odds ratios and 95\% confidence intervals were calculated to compare the completion rate between groups. Results Results are summarised in the table

Conclusion Consultants were more likely to achieve complete colonoscopy than previous trainees, who did not achieve $>90 \%$

\begin{tabular}{|c|c|c|c|c|c|}
\hline Group & Total colonoscopies & Complete procedures & Probability of completion & $\mathrm{OR}$ & $95 \% \mathrm{Cl}$ \\
\hline Consultant & 4439 & 4104 & 0.92 & $x$ & $x$ \\
\hline Previous trainees & 646 & 561 & 0.87 & 1.72 & $1.44-2.39$ \\
\hline Current trainees & 222 & 206 & 0.93 & 0.95 & $0.57-1.60$ \\
\hline
\end{tabular}

